Luca Arciero, Claudia Biancotti, Leandro D'Aurizio and Claudio Impenna (2009)

\title{
Exploring Agent-Based Methods for the Analysis of Payment Systems: A Crisis Model for StarLogo TNG
}

\author{
Journal of Artificial Societies and Social Simulation vol. 12, no. 12 \\ <http://jasss.soc.surrey.ac.uk/12/1/2.html>
}

For information about citing this article, click here

Received: 10-Mar-2008 Accepted: 12-Jun-2008 Published: 31-Jan-2009

8

\section{Abstract}

This paper presents an exploratory agent-based model of a real time gross settlement (RTCS) payment system. Banks are represented as agents who exchange payment requests, which are then settled according to a set of simple rules. The model features the main elements of a real-life system, including a central bank acting as liquidity provider, and a simplified money market. A simulation exercise using synthetic data of BI-REL (the Italian RTCS) predicts the macroscopic impact of a disruptive event on the flow of interbank payments. In our reducedscale system, three hypothetical distinct phases emerge after the disruptive event: 1) a liquidity sink effect is generated and the participants' liquidity expectations turn out to be excessive; 2) an illusory thickening of the money market follows, along with increased payment delays; and, finally 3 ) defaulted obligations dramatically rise. The banks cannot staunch the losses accruing on defaults, even after they become fully aware of the critical event, and a scenario emerges in which it might be necessary for the central bank to step in as liquidity provider.

Keywords:

Agent-Based Modeling, Payment Systems, RTCS, Liquidity, Crisis Simulation

\section{Introduction}

1.1

This paper presents an agent-based model of a real-time gross settlement system (RGTS), especially designed to simulate how interbank flows of liquidity evolve under critical conditions $\frac{[1]}{}$. We mainly seek to offer a methodological contribution, showing how the chosen modeling approach can be employed alongside other tools to study the generation and propagation of systemic risk in payment systems.

1.2

This topic is of growing interest due to the sharp increase in both cross-border payment activity and institutional attention to security and safety issues. In the context of RTCS systems, single large-value operations are settled one by one and immediately on condition that sufficient liquidity is held in participants' accounts, with several rules enforced in order to provide participants with adequate funds to execute payments throughout the day $[2]$. The system runs smoothly under ordinary conditions and is also well-suited to cope with common temporary liquidity shortages deriving from normal upswings of reserve stocks. However, mechanisms can be severely strained by negative financial or operational shocks affecting single participants or a group of them, resulting in danger to the entire system. This is why in the recent past oversight authorities (primarily central banks) have been promoting substantial research into how these situations arise, which parts of the system they affect most and in which order, with special reference to the most critical stage of the payment process: interbank settlement.

Our study stems from the latest developments in the simulation of RTGS systems, and seeks to build a knowledge base and an algorithmic representation for the core behavioral rules of participating banks. We develop a miniature agent-based framework that, while not exhaustive by any means, includes all major elements of the real world. Given its small scale and the limitations of the chosen software toolkit, the model presented in this paper should not be seen as readily relevant for operations. Rather, it should be taken as an exploration of the potential of agent-based methods in the analysis of payment systems. Compared with previous agent-based exercises, our model's main novelty is the explicit introduction of a money market, which plays a fundamental role in the evolution of the system after a critical event blocks or limits the activity of a participant. 
The paper is organized as follows. Section 2 describes the basic functioning of an RTGS system and highlights the importance of preventing systemic risk. Section 3 discusses literature related to the simulation of payment systems, and illustrates the advantages of the agent-based approach. Section 4 presents our model, section 5 accounts for the parameterization, and section 6 is devoted to the results. Section 7 concludes and outlines further work that is currently in progress.

\section{Real-time gross settlement systems and systemic risk: an overview}

2.1

In modern exchange economies, the smooth functioning of economic activity is heavily dependent on the reliability and the efficiency of payment systems. Cash transactions are steadily diminishing; consumers and firms generally settle their obligations through banks or other financial intermediaries, by means of instruments such as checks, money orders and electronic transfers. The intermediaries themselves initiate numerous payment flows for their own treasury operations or for other reasons.

2.2

The volume and value of transactions flowing through payment systems are ever-increasing, driven by financial liberalization and innovation, and by the globalization of the real economy. In the European Union alone, interbank payments amounted to 57 times annual GDP in 2005, up from 40 times at the end of the 1990s. Payment systems are increasingly interconnected, and their technical and functional complexity is high. Independent of the originator of a transfer and of the underlying instrument, each payment order enters an integrated process, going from the initial decision to transfer funds to a counterparty up to settlement in central bank money, i.e. an interbank flow of liquidity across the accounts banks hold at the central bank.

2.3

The costs associated with disruptions in payment systems are also climbing rapidly, since any interruption in the orderly functioning of the structure due to unexpected events is bound to affect an increasingly large number of transactions. The high degree of interdependence between participants facilitates the emergence of disruptive domino effects.

Central banks play a twofold role in settlement systems, which are public goods in nature, both as operators and overseers. They have an obvious interest in fostering financial stability, and in studying systemic risk so as to prevent disruptions where possible, and minimize their consequences otherwise.

2.5

Large-value operations represent a major potential source of systemic risk; to better keep it under control, starting from the second half of the 1990s most payment systems in the world have adopted the RTCS paradigm. [3]

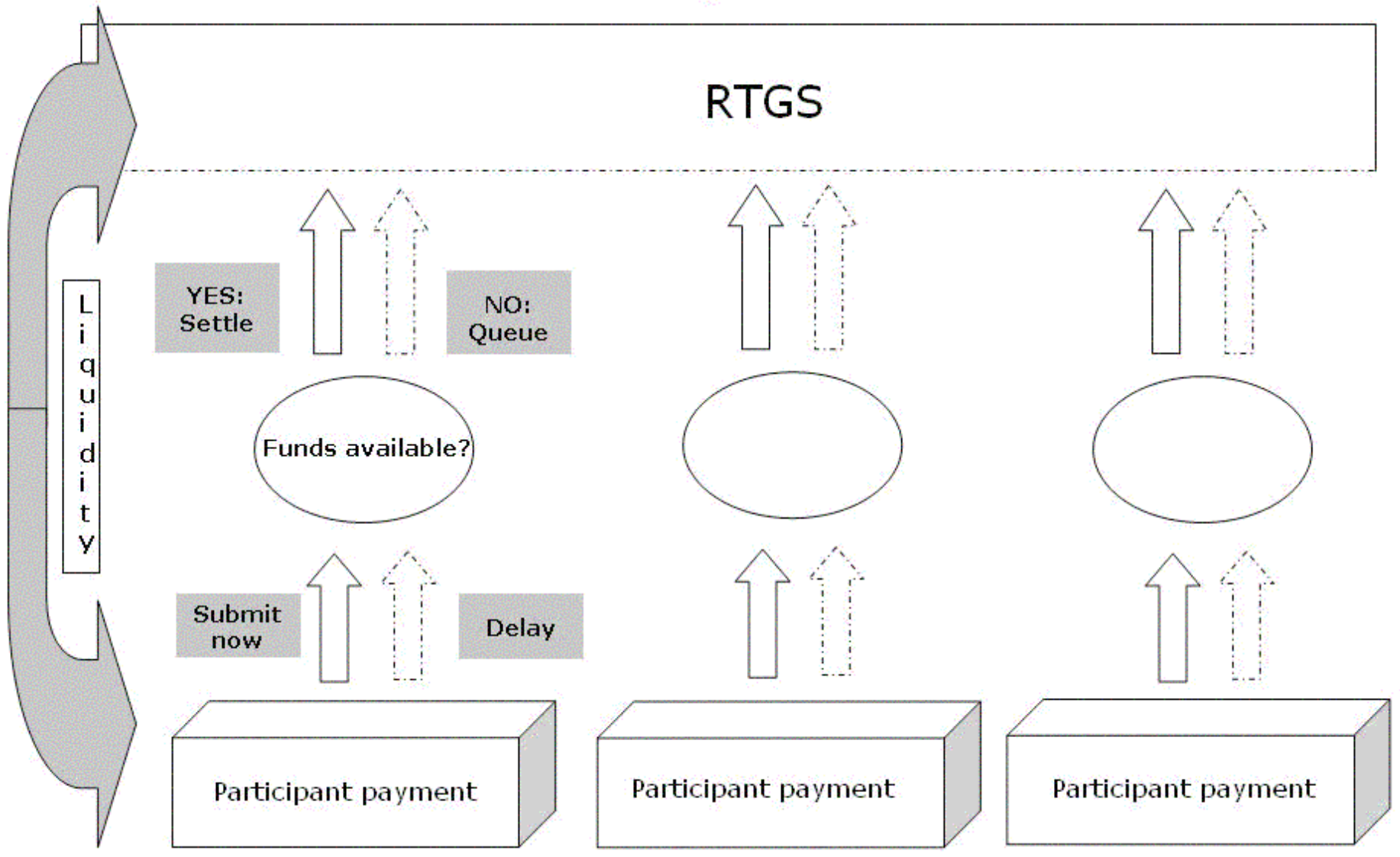


Figure 1. Basic functioning of an RTGS environment

2.6

Several intraday liquidity sources are available to banks to fund their outflows. Within an RTCS system, each participant normally relies on a continuous flow of incoming payments from its counterparties. Moreover, it can obtain intraday credit from the central bank; this entails a cost that may be either explicit, when the credit line is subject to a fee, or implicit, when the provision of funds is not priced but is conditional on the availability of collateral.

Alternatively, funds can be borrowed from other banks in unsecured or secured markets.

2.7

According to the actual and expected availability of each category of resources, banks make the strategic decision as whether to submit a payment promptly or delay it, thus affecting the overall time pattern of flows in the RTCS. In this respect, banks continuously face a trade-off between liquidity costs and delay costs. By releasing payments timely, banks satisfy customer and counterparty needs and benefit from a sound reputation, but they can incur high liquidity costs insofar as they need to borrow from the money market or the central bank. On the other hand, banks can play on the intraday dynamics of the money market more effectively by choosing to delay payments, at the expense of increased systemic risk and reputation uncertainty.

2.8

Once a payment is submitted to the system it is settled immediately if funds are available; otherwise, it is queued. The payment will be settled automatically once the shortage is overcome; banks can no longer affect the process at that point. It is worth noting that the tools and practices mentioned (delays, intraday credit, queues) are commonly used by system participants to cope with ordinary short-term liquidity swings. Questions need to be asked about the potential effects of an uncommon financial or technical shock on the functioning of an RTCS system. Such an event may induce prolonged, pathological illiquidity during the day, with potentially severe consequences for the smooth operation of the RTGS system, for financial stability and, ultimately, for economic activity.

\section{Advantages of the agent-based approach}

3.1

A well-established line of research uses traditional simulation techniques for the analysis and monitoring of settlement systems, with a view to the containment of typical credit, liquidity, and operational risks. Different tasks can be performed with different input data and simulation methods: historical data of payments submitted by banks can be used for "what if" analyses under different settlement mechanisms, while stochastic inputs can be used either for theoretical studies or for models aimed at extrapolating the consequences of particular behavioral assumptions on small-scale settings.

3.2

Along these theoretical lines, the Bank of Finland pioneered the construction of large-scale simulation models for settlement systems by building an ad hoc algorithm, where a deterministic stream of payments is accepted as an input and dealt with in a centralized manner under different hypotheses on the operating rules. Bank behavior is taken as given, or is made able to evolve in a predetermined manner. At present, the Bank of Finland simulator is widely used to evaluate the functioning of large-value payment systems under stressful conditions (see Bedford et al. 2005; Arnold et al. 2006; , Hellqvist and Koskinen 2005; Lublóy and Tanai 2007; Glaser and Haene 2007; Heijimans 2007). [4]

3.3

Compared with these earlier experiences, agent-based modeling has been introduced in this field only recently. Banks are seen as agents acting independently, and the system evolves as a result of their interaction. In some cases, intelligent adaptation to changing scenarios is allowed (seeFioretti 2005; Alentorn et al. 2006; Galbiati and Soramäki 2007).

3.4

The method appears to be particularly suitable for the problem for three reasons. First of all, it works well in terms of micro-foundations: in the short run, and especially where overnight activity is concerned, the behavior of commercial banks and of the central bank can be realistically described in terms of simple and consistent rules governing relatively straightforward decisions. The set of available strategies can be described in the language of game theory (Bech and Garrat 2003) and translated into algorithms with ease.

3.5

Secondly, agent-based models take the temporal dimension onboard fully; faced with a highly parallel, highly complex world, this feature is essential. Given a flow of due payments, banks mainly choose in which order to submit these payments to the system and how to obtain the liquidity necessary to meet obligations, subject to known constraints. Domino effects arising after a disruption can be properly described only if time is represented with a sufficient level of precision.

Last but not least, the flexibility of the agent-based approach constitutes an advantage when dealing with remarkably fast-changing environments, such as the financial one. Payment systems evolve; the banking sector consolidates, both domestically and internationally; some types of bank disappear, others gain prominence; occasionally, entire countries join a given 
system or withdraw from it; new financial instruments emerge; rules may change, at least partly. Traditional simulations need to be recalibrated, partly or entirely, whenever one of these discontinuities occur, as they often involve new analytical identifications of equilibria. On the contrary, agent-based models can be adapted efficiently with a relatively small number of alterations.

\section{The model}

4.1

Our model is built with StarLogo - The New Generation (TNG). $[5]$ We model a stylized version of the "plain-vanilla" RTGS system outlined in Section 2, excluding advanced liquidity management tools such as optimization and centralized queues. The model includes seven breeds of agents: banks, the central bank, payment requests, defaulted operations, interbank loan requests, crisis events and "craters", representing banks hit by them and consequently unable to perform any operation for a given period of time. One StarLogo second corresponds to one real-life minute. The logical flow of the model is outlined below, while the following Section discusses the parameterization.

During the setup phase, representing the opening of operations, banks are endowed with a starting level of cash and collateral. During the day, for every tick of the clock, each bank hatches a certain number of new agents, representing aggregations of all payment requests to be sent to a single counterparty at that moment. Once hatched, each payment request proceeds to cross the StarLogo terrain at fixed speed towards its destination.

For computational reasons, the RTCS system is treated as direct-debit based, with payments always requested by the payee. For greater realism compared with agent-based simulations that classify payments only on the base of their size and arrival time, payment requests in our model are assigned individual deadlines, ranging from "upon reception" (time-critical payments) to a certain number of minutes after reception. Amounts, deadlines and counterparties are determined through random draws, with features varying in accordance with desired scenarios. Upon arrival, a payment is queued until its deadline expires and triggers the settlement routine.

4.4

This submission rule allows for the implicit modeling of the settlement delay costs banks incur: for each operation, the delay cost is assumed to be a discontinuous function of time with a jump at the payment deadline and it is lower than liquidity costs until the deadline and higher afterward. [6]

4.5

From the moment a payment request is generated and until its settlement, its amount is incorporated in liquidity expectations for both the originating and the receiving bank. We therefore assume that at each moment banks are perfectly informed on all payment requests concerning them either as payer or payee; at time $t$ banks are able to calculate their future liquidity up to time $T$, where T- $t$ is the maximum lifespan of a payment request generated at $t$. The expectation is constantly updated as new payment requests enter the world.

When the settlement process starts, the intended payer tries to meet its obligation immediately. If the cash balance held at the moment is not sufficient, the bank tries to pledge collateral with the central bank, which provides liquidity based on $100 \%$ percentage. If collateral is also insufficient the bank tries to borrow on the money market ("short" bank), looking for a lending counterparty ("long" bank). The short bank $i$ randomly draws a potential counterparty $k$ from the pool of other banks. Bank $k$ agrees to lend if the condition $p^{i j}{ }_{t} \leq \mathrm{E}\left[\mathrm{L}^{k}\right.$ $T$ ] is met, where $p^{i j}{ }_{t}$ is the payment request, $j$ is the bank that originated it, and $E\left[\mathrm{~L}^{k} T\right]$ is the expected cash balance for $k$ at time T. In other words, the loan to cover $p^{i j}{ }_{t}$ is or is not granted on the basis of both present cash balances and future liquidity expectations, as determined by other outstanding payments initiated by or sent to the potential lender.

4.7

When a loan request is refused, the payment request $p^{i j} t$ "bounces", and the "short" bank looks for another lender. A counter keeps track of the number of bounces per request: when they exceed a certain threshold - a function of the number of possible available counterparties - the model flags the bank that has to settle the payment as unable to obtain sufficient funds from anyone. The request is cancelled and flagged as a defaulted obligation; its amount is recorded as an instance of insolvency for the "short" bank, and as a loss for the intended payee bank. Liquidity expectations for both ends of the transaction are adjusted accordingly. $[7]$

Disaster is simulated through the introduction of an agent of the "disruptive event" breed. A bank picked at random becomes totally inactive: it neither makes nor receives payment requests, nor does it operate in the interbank market as lender or borrower. Initially, i.e. in the first thirty minutes after the disruption has taken place, no agent is aware of it and all surviving banks continue their routine activity. A random process then makes the banks aware of disaster, with the probability of awareness increasing over time. Once a bank is aware, it stops requesting payments from the inactive bank, no longer considers it as a counterparty 
for loan requests, transforms all payment and loan requests pending towards it into defaulted obligations and updates losses and expected liquidity.

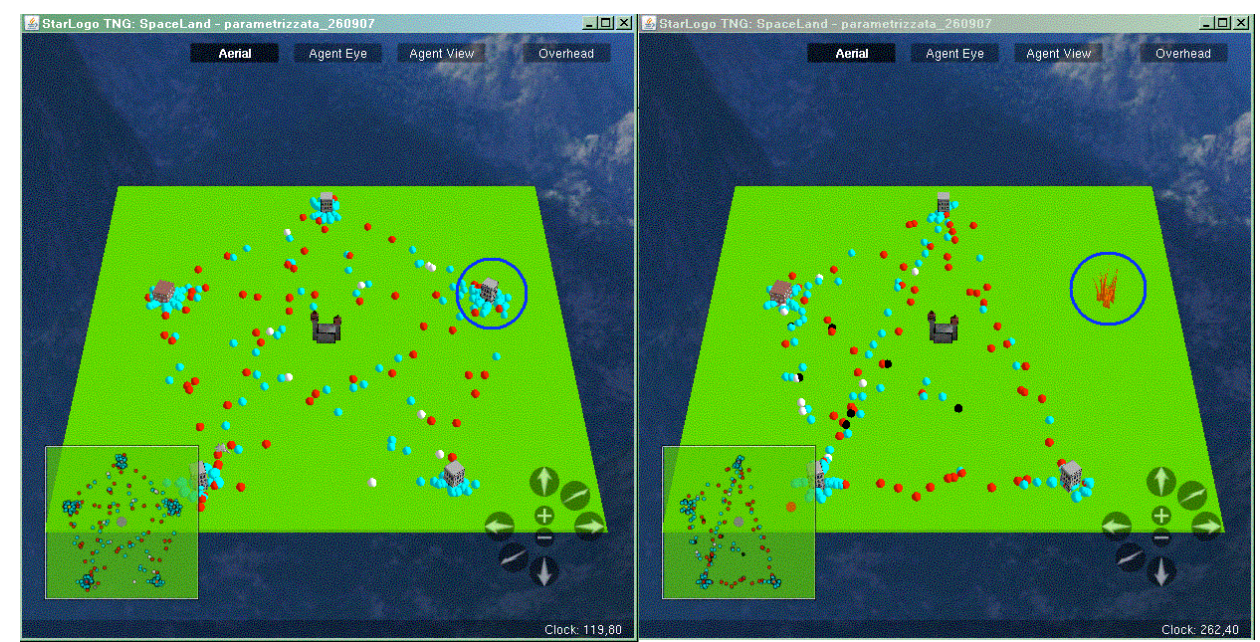

Figure 2. The simulated world before and after the critical event (The simulated world is depicted one second before (left) and 140 seconds after (right) a critical event hitting the circled agent. In the "after" scenario, enough time has elapsed since the disruption to allow all banks to exclude the inactive bank from the flow of payment requests, represented by colored spheres moving on the terrain.)

4.9

Depending on the simulated scenario, the central bank monitoring module may also be activated, although we do not use it in our simulation. When it is active, the central bank starts checking whether banks are delaying their payments beyond some pre-determined normal threshold. If a bank shows an excessive number of delays, the central bank supplies it with a sufficient amount of cash. The intervention routine stops when the number of delays returns below the threshold.

\section{Parameterization}

5.1

We use a set of summary statistics extracted from real data to calibrate the model. Data are drawn from the statistical archives that the Bank of Italy maintains in order to perform its

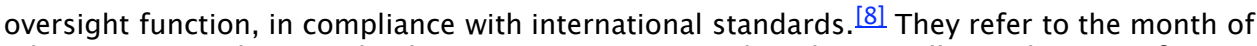
July 2007. More than 100 banks participate in BI-REL directly; we collapse them into five agents. .9$]$ The aggregation is necessary because the preliminary modeling step presented in the paper is aimed at capturing the essential traits of the agents' interactions, which can be best dealt with by focusing on a small number of agents. 10$]$ Each of our agents of the breed "banks" is therefore a "superbank" of sorts, incorporating banks that appear homogeneous in terms of payment traffic, opening balances, and collateral..[11]

Table 1 describes the five agents as they are in the real world. Superbanks 1 to 4 are aggregations of Italian banks, while superbank 5 is the aggregation of the Italian branches of foreign banks. The Bank of Italy collects elementary data for each payment channeled in the $\mathrm{BI}-\mathrm{REL}$ system, including information on the counterparties involved, the type of payment $[12]$ and the time at which it is submitted, settled or canceled. We transform this mass of data into a simplified archive, containing aggregate per-minute payment flows submitted by the five fictitious superbanks.

5.3

Payments involving individual banks belonging to the same superbank (intragroup flows), which amount to about 40 per cent of the total given in Table 1, are not considered. This does not present substantial drawbacks for the model, as most of these payments occur over the counter and are channeled through internal accounts rather than through BI-REL.

Data on cash balances refer to the fiat money banks hold at their RTCS accounts at the start of the operational day, while data on collateral are estimated on the basis of the maximum amount of collateral pledged at the central bank during the day.

Table 1: Main scenario (€ millions)

$\begin{array}{lllll}\begin{array}{l}\text { Agent } \\ \text { number payments } \\ \text { settled daily }\end{array} & \begin{array}{l}\text { Aarge } \\ \text { settled } \text { (\% of the } \\ \text { total) }\end{array} & \begin{array}{l}\text { Average end- } \\ \text { of-day } \\ \text { liquidity }\end{array} & \begin{array}{l}\text { Average end- } \\ \text { of-day } \\ \text { collateral }\end{array} \\ 1 & 18,384 & 3.0 & 5,302 & 2,648 \\ 2 & 22,669 & 6.6 & 3,583 & 3,947 \\ 3 & 13,718 & 3.1 & 4,780 & 2,766\end{array}$


apayments are defined "large" if their amount exceeds the $95^{\text {th }}$ percentile of the total distribution of flow values.

5.5

For our methodological purposes we just need to extract some stylized facts from the data observed and reproduce them within the simulation through a series of random draws.

5.6

We first observe that real-life payment distribution is highly concentrated

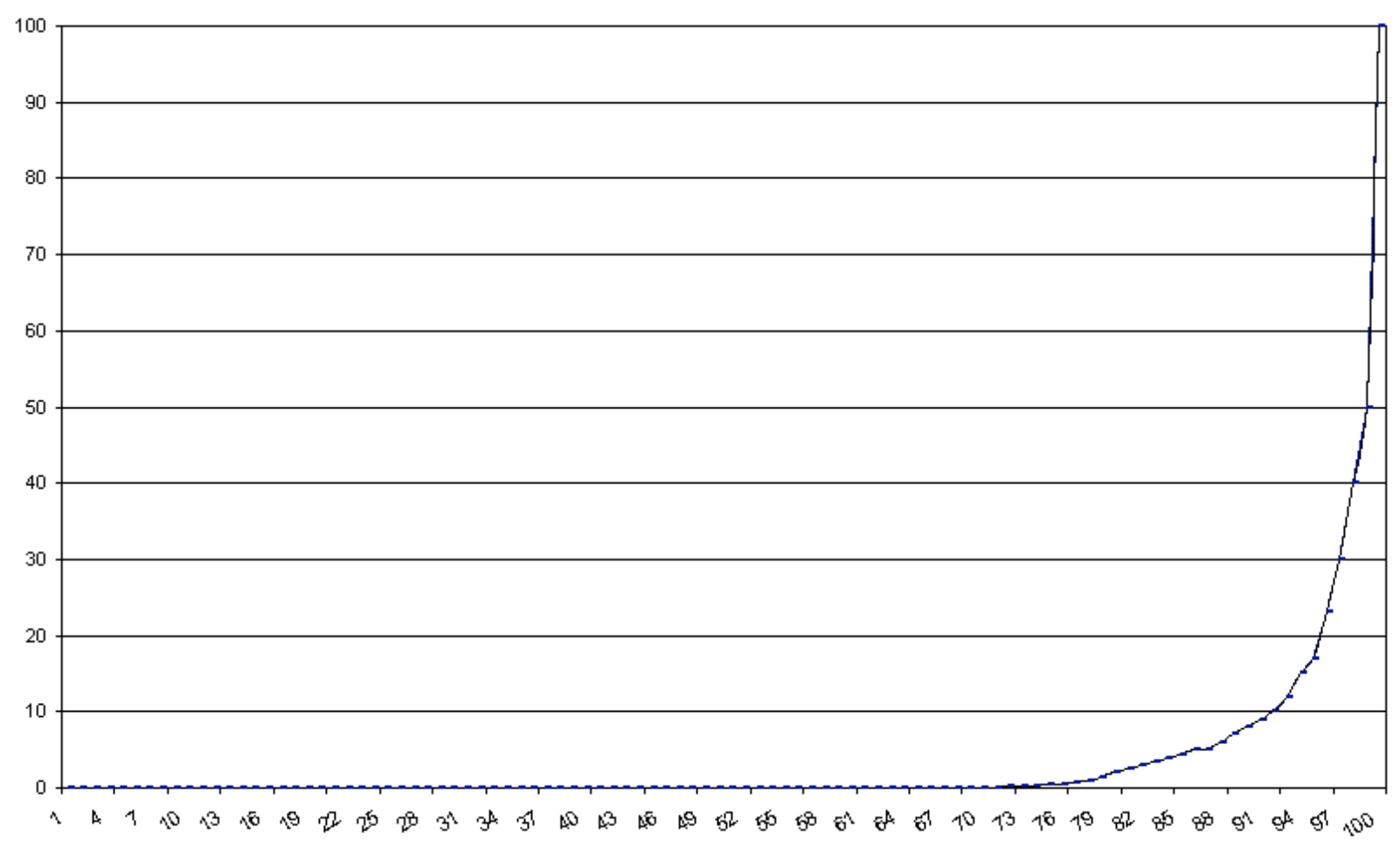

Figure 3. Concentration curve for payments submitted by the five superbanks

with payments larger than the $95^{\text {th }}$ percentile accounting for 85 per cent of the total amount exchanged and those above the 99 th percentile alone accounting for 50 per cent. The average payment (2.9 million euros) is therefore scarcely representative of the data mass, and such a high concentration makes kernel estimates of the underlying density function very unreliable. The relevant feature of the payment distribution is therefore that it is formed by a great mass of payments of relatively small values and an isolated cluster of huge ones; also that tends to be evenly spread among participants.

5.7

The daily amounts of collateral and cash show a high degree of variability and do not follow easily detectable patterns of correlation according to an exploratory analysis of the two series using the Johansen test of cointegration rank, not shown here for brevity. 


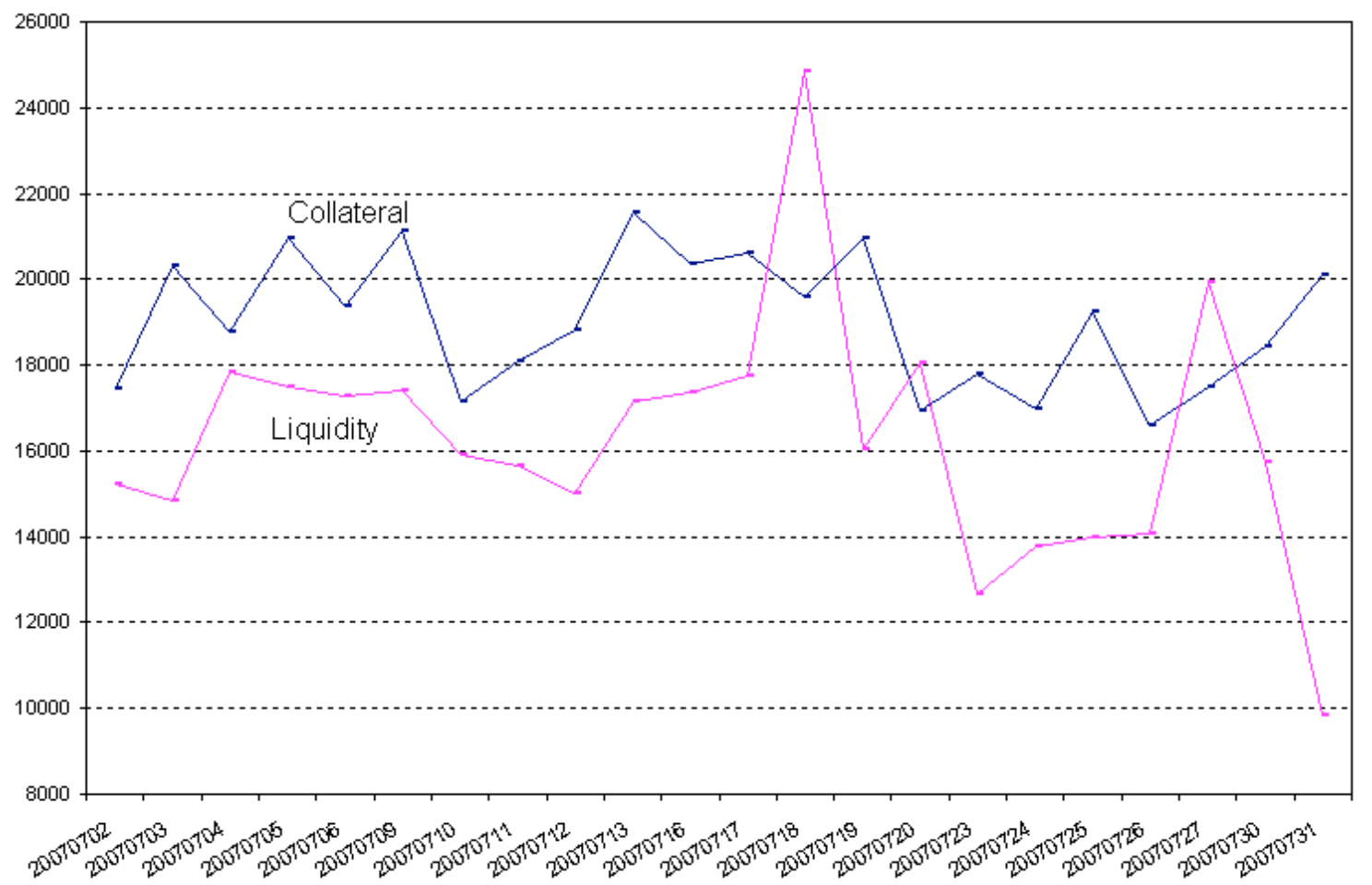

Figure 4. Total daily amounts of collateral and liquidity ( $€$ millions)

This could be explained by the fact that cash is also held to meet Eurosystem reserve requirements. Finally, the time series of daily payments is not correlated with either collateral or cash flow, which chimes with the fact that the vast majority of payments is settled through liquidity obtained in the course of the same day.

We reproduce these features as follows. Before the generation of payment flows, each fictitious system participant is initially endowed with liquidity and collateral. We first decrease the real daily amounts in our dataset by the share of payments generated by the payees not among the five units considered. These reduced amounts are further shrunk to take into account that they are also used to settle payments made by two participants belonging to the same superbank. We only simulate payments taking place between the superbank entities, and therefore neglect cross-border payments and those to and from the central bank.

5.9

For each of the five macro entities $i$, the means $\mu_{L i}, \mu_{C i}$ and the standard deviations $\mu_{L i}, \mu_{C i}$ of these final amounts of liquidity and collateral are computed. The starting levels of liquidity and collateral are approximately generated as draws from two uniform distributions defined in the intervals $\left[\mu_{L i}-\mu_{L i}, \mu_{L i}+\mu_{L i}\right],\left[\mu_{C i}-\mu_{C i}, \mu_{C i}+\mu_{C i}\right]$.

5.10

When it comes to generating payment flows, we emulate reality through a sequence of two per-minute draws from a uniform distribution for every participant: the first determines whether a payment request is to be submitted (reproducing the empirical frequencies) and the second, conditionally on the payment being submitted, decides its size class (small or large, with 98 per cent of submitted payments being small).

5.11

The final size of the payments is fixed by an additional random draw from a uniform distribution, centered on the appropriate class mean for payment size.

\section{Results}

6.1

Figures 5.1-5.5 depict the model predictions. $[13]$ The five panels represent the global evolution of liquidity levels, liquidity expectations (expressed as differences between current liquidity and liquidity as estimated after the settlement of all payment flows currently existing in the system), money market thickness, delays and losses incurred by banks in settlement activity. 

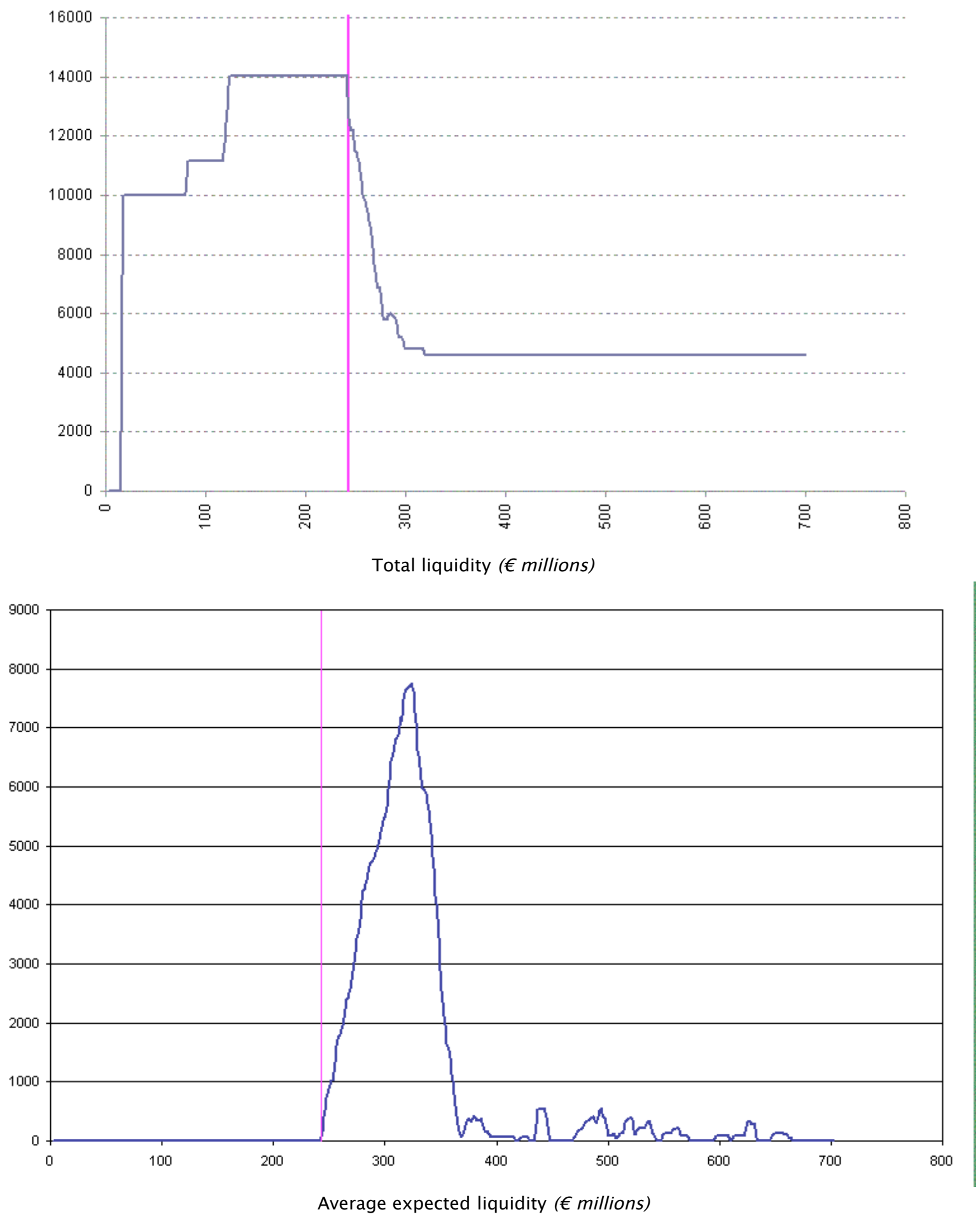

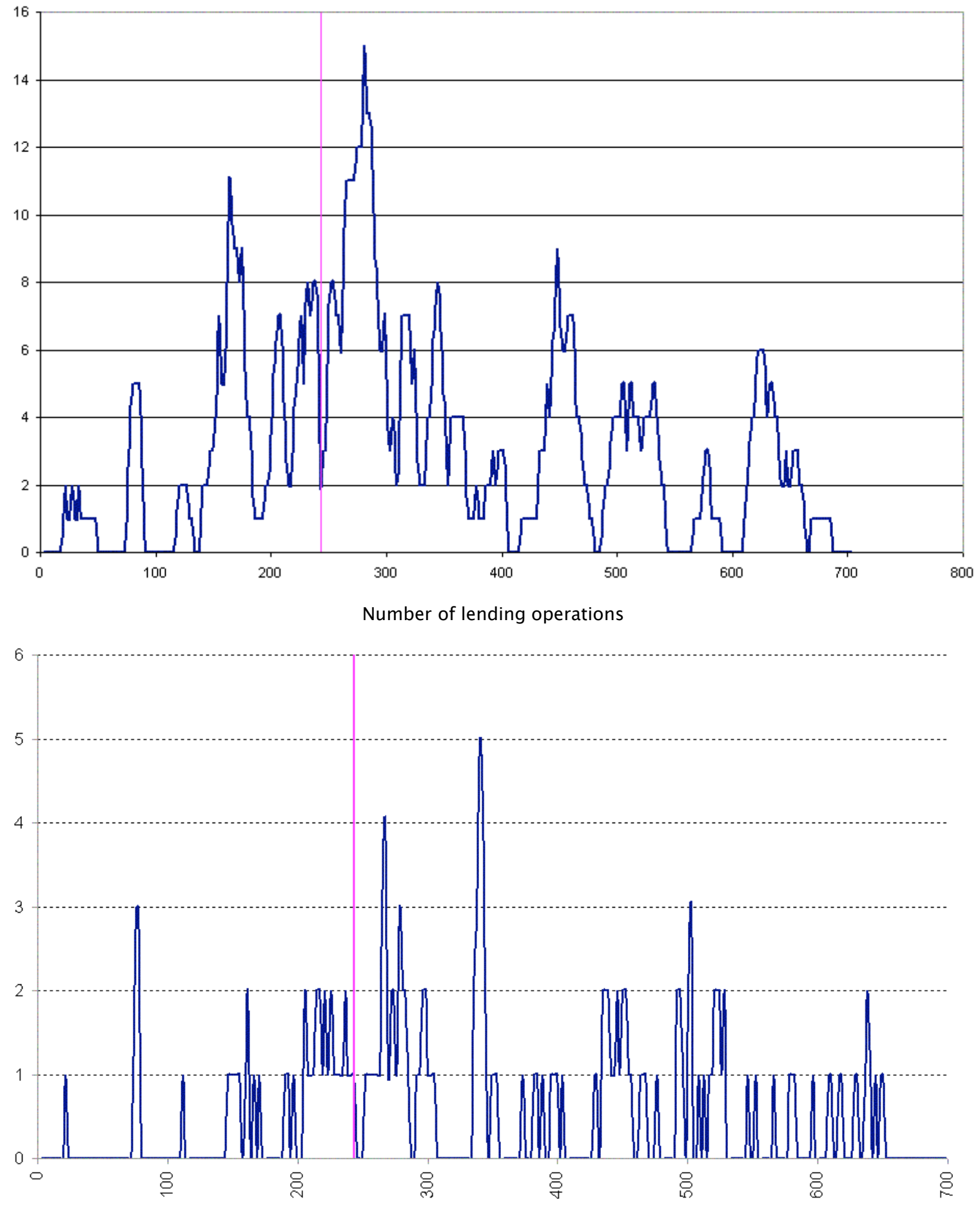

Number of delays 


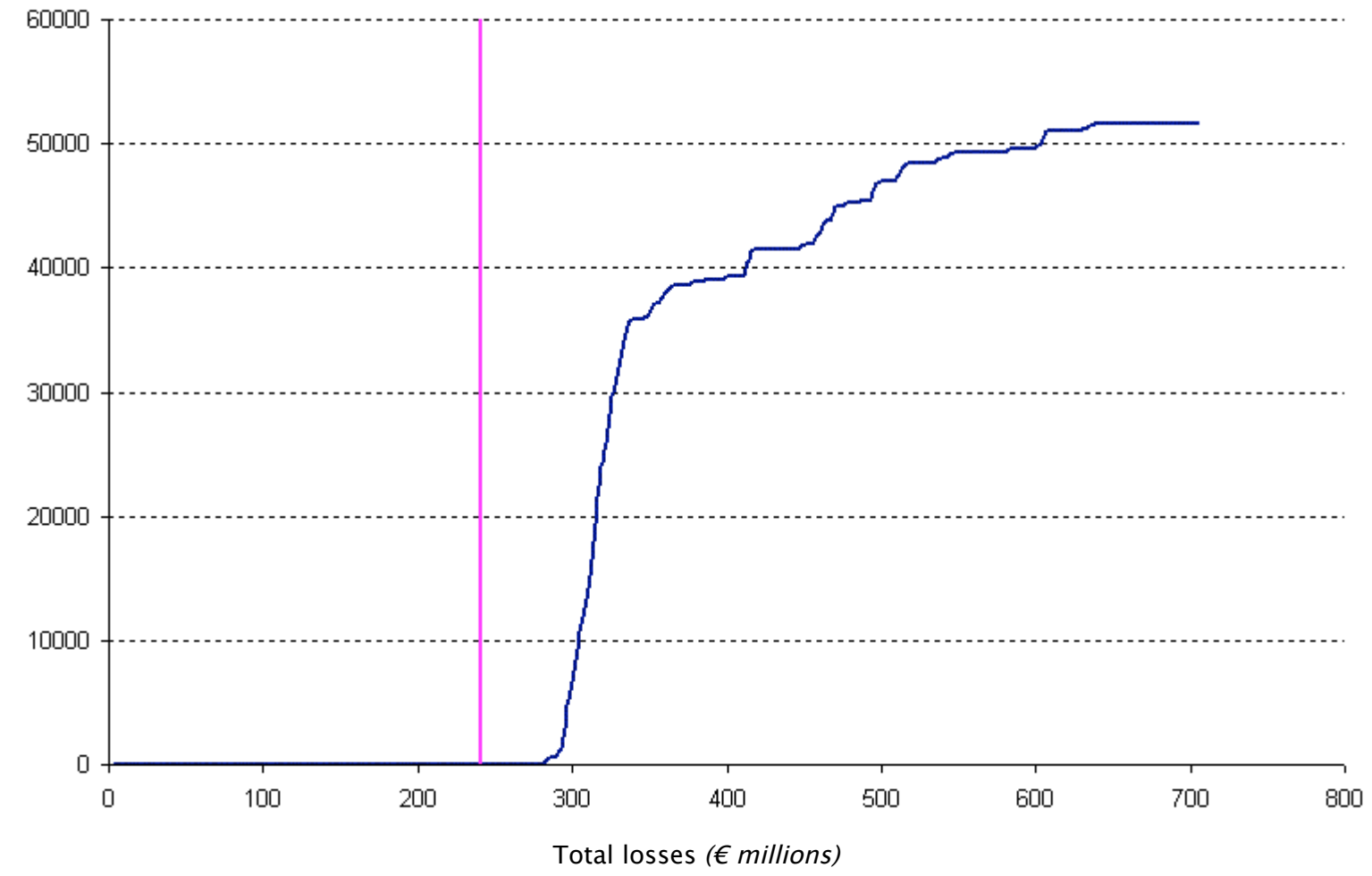

Figure 5. Time patterns of the relevant variables during the simulation (disruptive event indicated by the vertical line)

6.2

Under normal operating conditions, total liquidity follows quite a steady pattern, whereas the average expected liquidity is zero by definition, since we are modeling a closed world with five agents and what a single agent expects with the "plus" sign exactly corresponds to the same amount with "minus" sign for another. The banks' required liquidity is covered by money market interactions by a modest percentage, very close to the actual 5-7 per cent found for the Italian banking system.

6.3

When the disruptive event occurs and one of the banks is rendered unable to perform any transaction, the system evolves through three distinct phases. At first, when no agent is aware of what has happened, the average expected liquidity jumps from zero to large positive values, since the banks in operation keep on sending payment requests to the inactive one. They incorporate the future settlement of such requests in their expectations, but do not receive requests of the opposite sign in return. The regular counterbalancing mechanism for expectations fails; liquidity illusions emerge for all surviving banks.

6.4

This ushers in the second phase, marked by a sizable boom in money market transactions. Banks lack liquidity, because they do settle all the requests from the inactive bank that were pending at the time of the disaster, but their own requests towards it are not settled. After consuming their entire endowment of collateral in exchange for central bank money, they try to make up for the lack of liquidity by turning to the money market. Since loans are granted on the base of both current and expected liquidity, and the latter is artificially inflated, all banks are willing to lend to other banks: the thickness of the interbank market increases sharply and, as the actual liquidity fails to come in, delays accumulate. $[14]$

6.5

The third phase sets in as banks start to become aware of the disruptive event. One by one, they realize that one of their counterparties is not operational anymore and adjust their liquidity expectations accordingly. Money market activity slows down and losses accumulate.

6.6

Whether the system goes back to normal functioning, aside from the accumulated stock of defaulted obligations, depends on the relative impact of the disruptive event and its consequences compared with the total amount of liquidity in the system. According to our model, the system would not be able to react autonomously, i.e. without bailouts from the central bank, which were not implemented in this version of the simulation.

6.7

The results appear to be realistic. In this sense, they seem to confirm the robustness of the proposed methodology, although they are just a first stepping stone toward operational scenario analysis.

\section{Conclusions and further research}

7.1

In this paper we present an agent-based model of crisis simulation for a simplified RTCS 
payment system. The model's predictions approximate the macro-features of reality, showing the sequential intraday effects of an unexpected negative shock affecting a participant. The event first affects the liquidity stock and expectations of other participants. Then, the turbulence spills over to the interbank market and finally, when it has become visible in payment activity, necessitates adequate central bank intervention. Overall, the results shown above suggest that agent-based modeling can safely be included in the set of analytical tools that central banks use in their oversight activity and in the analysis of stability and resilience of financial systems.

7.2

The model is related to stress testing techniques, commonly employed by supervisory authorities and individual banks to quantify the effects of extreme financial or operational events. Stress testing methodologies share many traits with agent-based modeling, such as the focus on a given set of institutions, while behavior is governed by a pre-defined set of rules and generates feedback or "second round" reaction effects. Traditional stress tests may however put too little emphasis on market-wide conditions, being more focused on firmspecific levels, and accordingly they underestimate some liquidity risks originating in new financial products and techniques. Agent-based simulation may be especially suitable to offset these shortcomings, since it is flexible enough to allow for increasing complexities in the structure of financial networks such as settlement systems.

7.3

Our framework can be improved in several directions, so as to better reflect real RTCS and money market environments. At the time of writing, a larger model is under development, based on Pietro Terna's Swarm-Like Agent Protocol in Python (SLAPP; for further information, see the author's webpage at http://web.econ.unito.it/terna). Fostered by the encouraging results of the exercise described in this paper, and intended for scenario analysis of operational relevance, it is geared up for more realistic settings through some major changes. The payment submission process moves from a direct-debit to a credit-transfer based framework, where payments are submitted by the payer. There are as many agents as reallife participants in the Italian RTCS system; sources of uncertainty can be introduced by relaxing the independence assumption underlying payment generation and the hypothesis of common knowledge of environmental variables. Banks are assigned an end-of-day target in terms of cash balances, so as to mimic the inter-day liquidity management optimization pursued during the maintenance period for required reserves. The modeling of the money market overcomes most of the obstacles outlined in Section 4, also taking into account the lessons learned from the liquidity crisis of August 2007. The role of the central bank as an autonomous payer and payee is considered together with its function of liquidity supplier. The world outside BI-REL is considered in the form of an external agent that delivers stochastic shocks.

7.4

It is important to note that, where liquidity risk assessment is concerned, a deeper understanding of intraday dynamics is not the only use to which agent-based models can be put. An area currently commanding attention is, for example, that of strategic liquidity management, dealing with the way banks decide on the structure of maturity ladders, and how it can be affected by regulators seeking to minimize systemic risk. As a second example, agent-based techniques may shed light on the reasons behind the recent changes in the relative weight of exchanges on secured and unsecured markets, and help simulate the impact of yet-to-come technological, financial and regulatory innovation.

\section{Notes}

1 We would like to thank Paolo Angelini, Neville Arjani, Emilio Barucci, Lorenzo Cupido, Andrea Gerali, Marco Galbiati, Luigi Federico Signorini, Pietro Terna, attendees at seminars at the Bank of Italy, participants at the Agent2007 conference and two anonymous referees for useful suggestions. The development of the model benefited greatly from Claudia Biancotti's stay with the StarLogo development team at MIT: we especially want to acknowledge the contribution of Aidan Ho, Eric Klopfer and Daniel Wendel. All mistakes are ours. The opinions expressed in this paper are those of the authors, and should not be attributed to the Bank of Italy.

2 A comprehensive description of the design and activity of RTCS systems in most industrialized countries can be found in Bank for International Settlements (2005).

3 The alternative settlement mode to RTCS is the deferred net settlement (DNS) model, in which only net positions among banks are settled on their accounts at the central bank, usually once a day in the late afternoon. This system requires banks to hold less intraday liquidity, but exposes the payment system to higher risks of financial distress, since banks extend intraday credit to their debtors until the net settlement phase is successfully completed. A default by a bank unable to fund its net debit position could trigger a domino effect within the system.

${ }^{4}$ Beside this strand of studies based on a simulation approach, some authors have analyzed the effects of disruptive events from an econometric perspective. Among others, McAndrews and Potter (2002) use a panel approach to estimate how US banks participating in the US RTCS system Fedwire changed their strategy in term of payment submission during the 9/11 crisis. Payment systems can also be represented as a complex network, with banks as nodes and mutual liabilities/claims defining the arches; this kind of modeling is static, in the sense 
that the time dimension is not directly taken into account. Network theory has been exploited to study the main features of real interrelations among Austrian banks (Boss et al. 2004), making it possible to understand the system's level of concentration, i.e. whether few banks are responsible for the bulk of the links. The consequences of catastrophic events are simply modeled by removing a specific node and measuring the performances of the rest of the network.

5 Available for download at http://education.mit.edu/starlogo-tng/.

6 Though not game-theoretically founded, this rule satisfactorily reflects the fact that banks schedule a large share of outgoing payments according to intraday deadlines (cut-offs). These cut-offs can be agreed with customers, who initiate payments, or with the receiving bank, when the payment arises from interbank trades; see for example the guidelines of the Euro Banking Federation for money market-related payments. Alternatively, they might derive directly from the institutional setting, as in the case of payments related to monetary policy operations.

7 Our representation of the money market is partial. First of all, there are no prices, which equates to assuming that the overnight interest rate on the market is constant throughout the day, and equal for all pairs of counterparties. Empirical evidence points in a different direction, highlighting how the price of funding decreases during the day (Furfine 2001; Baglioni and Monticini 2007), how banks discriminate among counterparties (lori et al.2008; Furfine 2003), and how the cost of overnight funding might depend, among other factors, on the perceived creditworthiness of each participant (Nobili and Picillo 2008). We also overlook the existence of repo markets as an alternative to unsecured funding, in spite of their everincreasing importance. These aspects have to be taken onboard in any model that aims at being operationally relevant. In the case of the exercise presented here, however, we merely aim at giving a macro-level sketch of how interbank transactions might be instrumental in the transmission of external shocks to settlement activity in the short run. The very introduction of a money market innovates with respect to previous agent-based models; for the time being, we do not focus on interest rate dynamics.

8 Notably, the 2001 BIS Core Principles for Systemically Important Payment Systems and the consequent framework of the European System of Central Banks.

9 The five agents do not include non-banks, i.e. the central bank, central securities depositories and other clearing agents, as well as banks not directly participating in BI-REL, but using EU-RTCS systems linked to it.

10 Some tests on standard systems have shown that the current version of StarLogo TNG cannot handle the real-life number of banks. While the simulation still runs if there are 20 banks, the informational gain does not appear to be substantial enough to offset the losses in terms of speed and stability of the simulation.

11 The BI-REL system is, however, quite concentrated; two superbanks, for example, correspond to actual single banking groups, with a high level of internal coordination in the payment system.

12 Broadly speaking, the "type of payment" attribute indicates whether a transaction is initiated by a bank customer or stems from the bank's own activity in the financial market.

13 The results presented here refer to a single "frozen" run that should be taken as representative of the typical behavior of the system, since it was quite similar to many other runs we did not keep. At the time of writing, the StarLogo TNG platform did not allow for automatic averaging of the results of multiple runs, and did not track the changes in random seeds over the course of a single simulation sessions or over different sessions. The features for the preservation of results were in an experimental phase, and hence not entirely stable.

14 Note that this result is a consequence of the joint absence from the model of money market prices and general trust levels. Should these variables be included, a disruptive event might well result in a contraction of money markets, along the lines of what happened during the subprime crisis.

\section{References}

A. ALENTORN, S. Markose, S. Millard and J. Yang, 2006, "Designing large value payment systems: an agent-based approach", mimeo.

J. ARNOLD, M. L. Bech, W. E. Beyeler, R. J. Glass and K. Soramäki, 2006, "The topology of interbank payment flows", Federal Reserve Bank of New York, Staff Report 243.

BANK for International Settlements, 2005, "New developments in large-value payment systems", Committee on Payment and Settlement Systems.

A. BAGLIONI and A. Monticini, 2007, "The intraday price of money: evidence from the e-MID market", mimeo, available from http://www.ssrn.com.

M. BECH and R. Garratt, 2003, "The intraday liquidity management game", Journal of Economic Theory, 109: 198-219. 
P. BEDFORD, S. Millard and J. Yang, 2005, "Analysing the impact of operational incidents in large-value payment systems: a simulation approach", in H. Leinonen (ed.), Liquidity, risks and speed in payments and settlement systems - a simulation approach, Bank of Finland Studies, E:31.

M. BOSS, H. Elsinger, M. Summer and S. Thurner, 2004, "Network topology of the interbank market", Quantitative Finance, 4: 677-684.

G. FIORETTI, 2005, "Financial fragility in a basic agent-based model", mimeo.

C. FURFINE, 2001, "Banks as monitors of other banks: evidence from the overnight federal funds market", Journal of Business, 74: 33-57.

C. FURFINE, 2003, When is Inter-Transaction Time Informative?, Federal Reserve Bank of Chicago Working Paper No. 2003-04.

M. GALBIATI and K. Soramäki, 2007, "A competitive multi-agent model of interbank payment systems", mimeo.

M. GLASER and P. Haene, "Simulation of participant-level operational disruption in Swiss Interbank Clearing", 2007, presentation given at the 5th simulator workshop in Helsinki, http://www.bof.fi/en/rahoitusmarkkinat/kehityshankkeet/BoF-PSS2/seminar07.htm

R. HEIJIMANS, 2007, "Stress simulations: a Dutch case", presentation given at the 5th simulator workshop in Helsinki,

http://www.bof.fi/en/rahoitusmarkkinat/kehityshankkeet/BoF-PSS2/seminar07.htm.

M. HELLQVIST and J. Koskinen, 2005, "Stress testing securities clearing and settlement systems using simulations", in Leinonen, H. (ed.), Liquidity, risks and speed in payments and settlement systems - a simulation approach, Bank of Finland Studies, E:31

G. IORI, G. De Masi, O. Precup, G. Gabbi and G. Caldarelli, 2008, "A Network Analysis of the Italian Overnight Money Market", Journal of Economic Dynamics and control, 32: 2008.

A. LUBLY and E. Tanai, 2007, "Operational disruption and the Hungarian RTGS system VIBER", presentation given at the 5 th simulator workshop in Helsinki, http://www.bof.fi/en/rahoitusmarkkinat/kehityshankkeet/BoF-PSS2/seminar07.html.

J. MCANDREWS and S. M. Potter, 2002, "Liquidity effects of the events of September 11, 2001", FRBNY Economic Policy Review, 59-79, November.

A. NOBILI and C. Picillo, 2008, "Cost of funding in the money market and banks' creditworthiness: evidence from e-MID", mimeo, Bank of Italy.

Return to Contents of this issue

(ㄷ. Copyright Journal of Artificial Societies and Social Simulation, [2009] 